\title{
Analysis on Random Access Process of Single Carrier FDMA System
}

\author{
Doo Hwan Lee \\ Graduate School of the Frontier Informatics, The \\ University of Tokyo \\ leedh@mlab.t.u-tokyo.ac.jp
}

\author{
Hiroyuki Morikawa \\ Graduate School of the Frontier Informatics, The \\ University of Tokyo \\ mori@mlab.t.u-tokyo.ac.jp
}

\begin{abstract}
In this paper we analyzed random access process of single carrier FDMA (SC-FDMA) system, which is one of uplink candidates in 3GPP long term evolution. In random access process each user equipments (UE) transmits randomly selected random access code and the Node B detects each UE's random access try by code identification. It is conducted by exploiting the cross-correlation property of random access code. Moreover, round trip time (RTT) is also estimated during random access detection process for uplink time synchronization utilizing the auto-correlation of random access code. This paper proposed random access detection scheme which adopts the frequency domain RTT estimation and the time domain code identification. The performance of random access of SC-FDMA system highly depends on correlation properties. However, they are susceptible to channel characteristics in the multipath fading channel environments. Thus, this paper analyzed the performance of random access in the multipath fading channel. Results of our analysis will be beneficial as the guideline in designing SCFDMA based random access system.
\end{abstract}

\section{Categories and Subject Descriptors}

C.2.1 [Computer-Communication Networks]: Network Architecture and Design-Wireless communication; C.4 [Performance of Systems]; I.6 [Simulation and Modeling]

\section{General Terms}

Algorithms, Performance, Design

\section{Keywords}

SC-FDMA, LTE, Random access

\section{INTRODUCTION}

Recently single carrier FDMA (SC-FDMA) has drawn much attention and it is adopted as one of uplink candidates in 3GPP long term evolution (LTE) [1-3]. SC-FDMA exploits single carrier modulation and frequency domain equalization. It has inherent low peak-to-average power ratio (PAPR) while having

Permission to make digital or hard copies of all or part of this work for personal or classroom use is granted without fee provided that copies are not made or distributed for profit or commercial advantage and that copies bear this notice and the full citation on the first page. To copy otherwise, to republish, to post on servers or to redistribute to lists, requires prior specific permission and/or a fee. WICON 2007, October 22-24, Austin, USA

Copyright (C) 2007 978-963-9799-12-7

DOP 10.4108/wicon.2007.2092 similar system complexity with OFDMA [4-5]. Thus, SC-FDMA is regarded as an alternative to OFDMA which suffers from high PAPR. In particular, SC-FDMA has a merit in terms of transmit power efficiency in uplink system.

In SC-FDMA based uplink system such as 3GPP EUTRA LTE, a new network entry is performed by contention-based random access. Some of channel resources are allocated for random access and new UEs, which are willing to join the network, transmit random access signal simultaneously. Since random access channel is granted only for initial network entry UEs, existing UEs' traffics do not interfere with the random access signal. However, other random access UEs' interferences exist in the multiple random access scenarios. The contention resolution among multiple UEs is carried out in the code domain utilizing the correlation property of random access code.

There are a few researches on analyzing the OFDMA based random access [6-10]. However, few researches have been conducted regarding SC-FDMA based random access since SCFDMA is relatively new technology. Thus, it is necessary to study on SC-FDMA based random access process including random access detection scheme and its performance analysis. Although a lot of inherent similarities exist between SC-FDMA and OFDMA, random access detection scheme of OFDMA is hard to be applied to that of SC-FDMA directly. This derives from the fact that random access code of OFDMA is applied in the frequency domain while that of SC-FDMA is applied in the time domain.

This paper proposes SC-FDMA random access detection scheme, which adopts the frequency domain multi-user resolution and the time domain uplink synchronization. Furthermore, the performance of random access is analyzed. The performance of random access highly relies on the correlation property of random access code, which is prone to be affected by channel characteristics. The performance degradation is much severe in the multipath fading channel. The previous researches [6-10] only adapted the benign channel. However it is necessary to consider the unfavorable channel environment to investigate the lower bound of system performance. Thus, the performance analysis will also consider multipath fading channel environment.

The remainder of this paper is organized as follow. Section 2 provides a brief review of SC-FDMA random access process and Section 3 describes the system model and proposed random access detection scheme. Performance analysis and numerical results will be given in Section 4. Section 5 will conclude this paper. Finally, Appendix will be provided for the analytical analysis. 

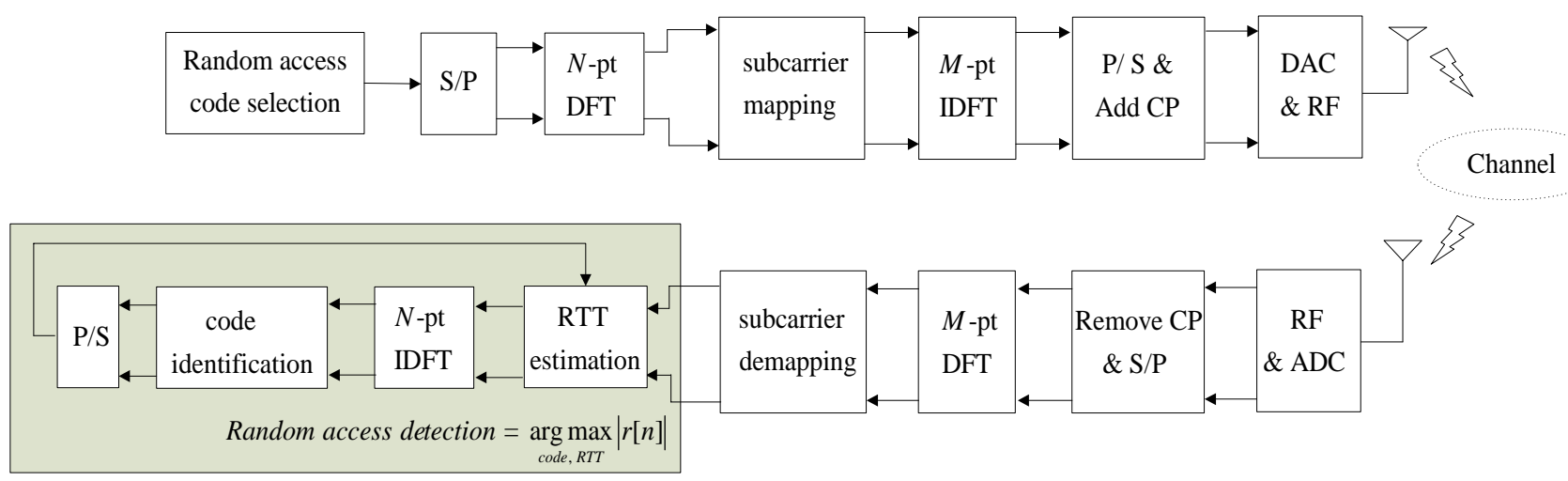

Fig. 1 Transmitter and receiver structure of SC-FDMA random access system.

\section{BACKGROUND}

\subsection{Random Access Process}

Random access procedure is classified into synchronized random access and non-synchronized random access. The former is defined for synchronized UE's usage such as bandwidth request. The latter is used when UE uplink is not synchronized or loses synchronization. Initial network entry function is performed based on non-synchronized random access. This paper will focus on non-synchronized random access to analyze the wireless initial access system. Without loss of generality random access will refer non-synchronized random access in this paper.

The Node B shall schedule random access channel periodically in order to provide wireless multiple access opportunity. For the initial network entry, a UE transmits randomly selected random access code through the scheduled random access channel. Multiple UEs may transmit their random access code simultaneously through the same channel. In this case, multi-user resolution is achieved by the random access code identification. The Node B monitors the random access channel to detect UE's transmission. Random access detection process consists of random access code identification and RTT estimation. Once the Node B detects UE's random access try successfully, it notifies the UE of the success message with RTT estimation result. Then, the UE synchronizes uplink timing with RTT information and proceeds further communication with the Node B.

\subsection{Random Access Code}

To provide satisfactory multiple access performance random access code has to have good cross-correlation and autocorrelation properties. Orthogonality of multiple UEs is maintained by the cross-correlation property and RTT is estimated by exploiting the auto-correlation property. Numerous codes are suggested and analyzed for the wireless uplink random access system [11-13]. Among them Zadoff-Chu code is chosen for our analysis since it is adopted in 3GPP LTE uplink system. Zadoff-Chu code the special case of Chirp-like polyphase sequences having the superb correlation property. In particular, the Crest factor of Zadoff-Chu code is maintained minimized regardless of the length of code, which corresponds to the philosophy of SC-FDMA system. Zadoff-Chu code is defined as Eq. (1),

$$
c[n]=\left\{\begin{array}{l}
\exp \frac{j H \pi n^{2}}{N}, \quad n=0,1, \ldots, N-1, \text { for } N \text { even, } \\
\exp \frac{j H \pi n(n+1)}{N}, n=0,1, \ldots, N-1, \text { for } N \text { odd, }
\end{array}\right.
$$

where $N$ is length of code and $H$ is the integer code index prime to $N$. For the simplicity $N$ is assumed to be even in this paper.

\section{SYSTEM MODEL}

This section describes the system model including the transmitter, the channel, the receiver, and the proposed detection scheme in SC-FDMA random access system. The overall structure is given in Fig. 1

\subsection{Random Access Process}

Consider an SC-FDMA system with total $\mathrm{M}$ subcarriers and $\mathrm{N}$ random access subcarriers. The $m^{\text {th }}$ UE which is willing to join the network selects the integer code index $H_{m}$ randomly. It obtained the previous knowledge on the basic physical parameters such as the number of random access subcarriers and the location of random access channel from the Node B's broadcast. The $m^{\text {th }}$ UE generates random access code $c_{m}[n]$ by Eq. (1) and they are converted to parallel and fed into $N$-point DFT block. Then the $k^{\text {th }}$ DFT output of the $m^{\text {th }}$ UE's random access signal is described as Eq. (2) in the frequency domain.

$$
S_{m}\left(f_{k}\right)=\sum_{n=0}^{N-1}\left(\exp \frac{j H_{m} \pi n^{2}}{N} \cdot \exp \frac{(-j 2 \pi k n)}{N}\right), k=0,1, \ldots, N-1
$$

DFT performed random access signals are mapped to subcarriers by distributed manner or localized manner. In distributed mapping scheme, which is called as interleaved FDMA (IFDMA), DFT outputs are allocated to non-continuous subcarriers over the entire uplink bandwidth. On the other hand, in localized mapping 
scheme, which is called as localized FDMA (LFDMA), DFT outputs are allocated to continuous subcarriers. In general, IFDMA is used to exploits channel diversity since each subcarrier experiences independent channel environment and LFDMA is used for modulation gain in the benign channel. After subcarrier mapping, random access signal both schemes are given as below.

$$
\begin{gathered}
\widetilde{S_{m}}\left(f_{k}\right)_{\text {LFDMA }}= \begin{cases}S_{m}\left(f_{k}\right), & 0 \leq k \leq N-1 \\
0, & \mathrm{~N} \leq k \leq M-1,\end{cases} \\
\widetilde{S_{m}}\left(f_{k}\right)_{\text {IFDMA }}= \begin{cases}S_{m}\left(f_{k}\right), & k=Q \cdot a(0 \leq k \leq N-1) \\
0, & \text { otherwise, }\end{cases}
\end{gathered}
$$

where $Q$ is spreading factor of IFDMA and $a$ is an integer satisfies $0 \leq a \leq N-1$. Subcarrier mapped random access signals are fed into $M$-point IDFT block and converted to serial. Cyclic prefix (CP) is added to prevent inter block interference and transmitted to the Node B after some RF operations. Transmitted random access signal is finally described as Eq. (4) in the time domain. In Eq. (4) $l$ equals to $(N \cdot a+b) . a$ and $b$ are integers which satisfy $0 \leq a \leq N-1$ and $0 \leq b \leq Q-1$, respectively.

\subsection{Channel}

$\mathrm{N}$-ary multipath Rayleigh fading channel model is adpoted in this paper. The $m^{\text {th }}$ UE's $i^{\text {th }}$ subcarrier channel impulse response (CIR) $h_{m, i}(t)$ is represented as

$$
h_{m, i}[t]=\sum_{r=0}^{N-1} A_{m, i}^{r} \alpha_{m, i}^{r} e^{j \phi_{m, i}^{r}} \delta\left(t-\tau_{m, i}^{r}\right)
$$

where $N$ is the number of multipath. $A_{m, i}^{r}, \alpha_{m, i}^{r}, e^{j \phi_{m, i}^{r}}$, and $\tau_{m, i}^{r}$ are the attenuation factor, random magnitude, random phase and multipath delay of the $r^{\text {th }}$ delay path, respectively. $A_{m, i}^{r}$ and $\tau_{m, i}^{r}$ highly depend on channel environments such as geometry and user's mobility. They are obtained from multipath profiles. $\alpha_{m, i}^{r}$ and $\phi_{m, j}^{r}$ are independent and identically distributed Rayleigh and uniform random variables, respectively. Probability density functions (PDF) of $\alpha_{m, i}^{r}$ and $\phi_{m, i}^{r}$ are given as

$$
\begin{aligned}
& f_{\alpha_{m, i}^{r}}\left(\alpha_{m, i}^{r}\right)=\frac{\alpha_{m, i}^{r}}{\left(\sigma_{m, i}^{r}\right)^{2}} \exp \left(\frac{-\left(\alpha_{m, i}^{r}\right)^{2}}{2\left(\sigma_{m, i}^{r}\right)^{2}}\right), \quad\left(0 \leq \alpha_{m, i}^{k}<\infty\right), \\
& f_{\phi_{m, i}^{r}}\left(\phi_{m, i}^{r}\right)=1 / 2 \pi, \quad\left(0 \leq \phi_{m, i}^{r}<2 \pi\right),
\end{aligned}
$$

where $\left(\sigma_{m, i}^{r}\right)^{2}$ is the second momentum of in-phase and quadrature components parts of the $i^{t h}$ subcarrier.

\subsection{Receiver}

The received random access signal is shown as a circular convolution form between the transmitted signal and CIR as below.

$$
r_{m}[l]=\left(s_{m}\left[l-l_{m}^{R T T}\right] * h_{m}[l]\right)_{M}+n[l],
$$

where $n[l]$ is AWGN with variance $N_{o} / 2$ and $l_{m}^{R T T}$ is RTT of the $m^{\text {th }}$ UE. CP plays a role as converting the linear convolution to the circular convolution provided that $\mathrm{CP}$ is longer than RTT plus maximum multipath delay.

The Node B performs the RF operations such as ADC with the received random access signal. Subsequently, CP removal and parallel conversion are followed and the output signals are fed into $M$-point DFT blocks. DFT-outputs are demapped into $N$ frequency band by distributed method or localized method. The demapped random access signal after DFT operation is described in the frequency domain as below.

$$
\begin{aligned}
& R_{m}\left(f_{k}\right)_{\text {LFDMA }}=\sum_{k=0}^{N-1}\left[S_{m}\left(f_{k}\right) \cdot H_{m}\left(f_{k}\right) \cdot \exp \left\{j 2 \pi f_{k}\left(l_{C P}-l_{m}^{R T T}\right)\right\}\right]+N\left(f_{k}\right), \\
& R_{m}\left(f_{k}\right)_{I F D M A}=\sum_{k=0}^{N-1}\left[S_{m}\left(f_{k}\right) \cdot H_{m}\left(f_{Q k}\right) \cdot \exp \left\{j 2 \pi f_{Q K}\left(l_{C P}-l_{m}^{R T T}\right)\right\}\right]+N\left(f_{Q k}\right) .
\end{aligned}
$$

$$
\begin{aligned}
& S_{m}[l]_{L F D M A}=\frac{1}{M} \sum_{k=0}^{M-1}\left(\widetilde{S_{m}}\left(f_{k}\right)_{L F D M A} \cdot \exp \frac{j 2 \pi k n}{M}\right)=\left(\begin{array}{ll}
\frac{1}{Q} \exp \frac{j H_{m} \pi n^{2}}{N}, l=0,1, \ldots, M-1, & \text { when } b=0 \\
\frac{1}{Q} \cdot \frac{1}{N} \sum_{n=0}^{N-1}\left(\exp \frac{j H_{m} \pi n^{2}}{N} \cdot \frac{1-\exp \{j 2 \pi(a-n+b / Q)\}}{1-\exp [j 2 \pi\{(a-n) / N+b / Q N\}]}\right), \text { when } b \neq 0 .
\end{array}\right. \\
& S_{m}[l]_{I F D M A}=\frac{1}{M} \sum_{k=0}^{M-1}\left(\widetilde{S_{m}}\left(f_{k}\right)_{\text {IFDMA }} \cdot \exp \frac{j 2 \pi k n}{M}\right)=\frac{1}{Q} \exp \frac{j H_{m} \pi(l \bmod n)^{2}}{N}, l=0,1, \ldots, M-1 . \\
& r_{m}[n]_{L F D M A}=\sum_{k=0}^{N-1}\left[c_{m}[n](\tilde{C}[n])^{*} \cdot H_{m}\left(f_{k}\right) \cdot \exp \left\{j 2 \pi f_{k}\left(l_{C P}-l_{m}^{R T T}-\Delta l\right)\right\}\right]+n^{\prime}[n], n=0,1, \ldots N-1, \\
& r_{m}[n]_{I F D M A}=\sum_{k=0}^{N-1}\left[c_{m}[n](\tilde{c}[n])^{*} \cdot H_{m}\left(f_{Q k}\right) \cdot \exp \left\{j 2 \pi f_{Q k}\left(l_{C P}-l_{m}^{R T T}-\Delta l\right)\right\}\right]+n "[n], n=0,1, \ldots N-1 .
\end{aligned}
$$




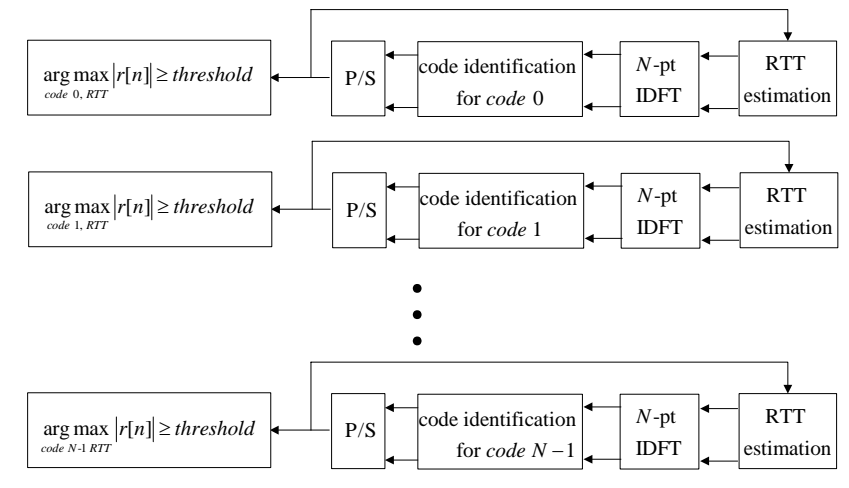

Fig. 2 Structure of multiple random access detection.

\subsection{Proposed Random Access Detection Scheme}

In this part, we propose the random access detection scheme which includes the frequency domain RTT estimation and the time domain random access code identification. The node $\mathrm{B}$ multiplies $\exp \left(-j 2 \pi f_{k} \Delta l\right)$ to each subcarrier of the received signal for the RTT estimation. Subsequently, N-point IDFT is performed. Then, the Node B correlates the IDFT outputs with complex-conjugated random access code $(\tilde{c}[n])^{*}$. Hereby, $r_{m}[l]$ is described as Eq. (9). $n$ '[n] and $n^{\prime \prime}[n]$ have statistically identical characteristics with $n[l]$ since they are simply phase transformed version of $n[l]$. This process is carried out for all possible $\Delta l$ and $\tilde{c}[n]$ since the Node B is unaware of $l_{m}^{R T T}$ and $c_{m}[n]$. Finally, the node $\mathrm{B}$ selects $\Delta l$ and $\tilde{c}[n]$ which have the maximum absolute value as shown in Eq. (10).

$$
\tilde{(C}[n], \Delta l)_{\text {selected }}=\underset{\tilde{c}[n], \Delta l}{\arg \max }|r[n]| .
$$

In the perfect channel, $|r[n]|$ will have the maximum value when $\tilde{c}[n]$ equals to $c_{m}[n]$ and $\Delta l$ equals to $\left(l_{C P}-l_{m}^{R T T}\right)$. However, the possibility of discordance exists in the multipath due to channel component $H_{m}\left(f_{k}\right)$ and $H_{m}\left(f_{Q k}\right)$.

\subsection{Multiple Access}

SC-FDMA random access provides wireless multiple access functionality as well. Multiple UEs selects random access code randomly and transmit them to the Node B simultaneously. Each UE's signal experiences different channel environments with different RTT and the received signal at the Node B is given as below.
Table 1. System Parameters of SC-FDMA random access

\begin{tabular}{ll} 
Frame length & $5 \mathrm{msec}$ \\
Sub-frame length & $0.5 \mathrm{msec}$ \\
Block size & $66.67 \mathrm{usec}$ \\
Cyclic prefix duration & $6.25 \mathrm{usec}$ \\
Number of random access & $72,84,96,108,120$ \\
subcarriers & 600 \\
Total number of subcarriers & 1024 \\
DFT size (samples) & $15 \mathrm{KHz}$ \\
Subcarrier spacing & $1.08, \quad 1.26, \quad 1.44, \quad 1.62$, \\
Random access bandwidth & $1.80 \mathrm{MHz}$ \\
& $10 \mathrm{MHz}$ \\
Total bandwidth & $0.5 \mathrm{Km}$ (Micro) \\
Cell size & 6 \\
Spread factor of IFDMA & \\
\hline
\end{tabular}

$$
r[l]=\sum_{m=0}^{I-1}\left(s_{m}\left[l-l_{m}^{R T T}\right] * h_{m}[l]\right)_{M}+n[l],
$$

where $I$ is the number of UEs which transmit random access signal simultaneously. Likewise the single user case, the Node B carries out RTT estimation and code identification of each UE. Fig. 2 depicts the structure of multiple random access detection. For each random access code, the Node B performs RTT estimation by peak detection by searching $l^{R T T}$ which maximizes the output. Then, the Node B obtains $(N \times 2)$ vectors which include the maximum output and corresponding $l^{R T T}$ for each random access code. The Node B compares the maximum output of each random access code with the predefined threshold. In case the maximum output of random access code $i$ is bigger than the threshold. The Node B determines that a certain UE transmits random access code $i$ and its RTT is estimated to the corresponding $l^{R T T}$ of the random access code $i$. In this way, the Node B is able to detect multiple UEs random access signal without knowledge of the number of UEs by multiple access detection. This blind detection method causes hardware burden as the number of random access code increases. Thus, the reasonable grouping of the random access code set is necessary.

\section{PERFORMANCE ANALYSIS AND NUMERICAL RESULTS}

\subsection{System Parameters}

System parameters of SC-FDMA random access are chosen from [1] and [14]. They provide various set of system parameters. Among them most appropriate parameters are chosen for our analysis. Details are given in Table 1. Frame length which contains 10 sub-frames is set to 5 msec. In TDD system, downlink and uplink transmission opportunities are given every sub-frame ( $0.5 \mathrm{msec})$ while random access channel is allocated every frame ( $5 \mathrm{msec}$ ). One block size is defined as $66.67 \mathrm{usec}$ which refers subcarrier spacing is $15 \mathrm{KHz}$. Number of random access subcarriers are set to 72, 84, 96, 108, and 120 with corresponding random access bandwidth 1.08, 1.26, 1.44, 1.62, and $1.80 \mathrm{MHz}$. DFT size $M$ is set to 1024 and DFT one sample interval is 0.065 
usec. Spread factor of IFDMA is defined as 6 and one sub-carrier spacing of IFDMA is $90 \mathrm{KHz}$. Micro cell is adopted and cell size is set to $0.5 \mathrm{~km}$. To reduce the discrepancy between simulation and real world, Rural and Urban channel models are adopted from [15].

\subsection{Metrics of Performance Analysis}

To evaluate the performance of SC-FDMA random access two metrics are defined as below.

(1) Random access detection miss rate $\left(P_{M}\right)$.

(2) RTT estimation error $\left(P_{E}\right)$.

$P_{M}$ and $P_{E}$ refer the probability that the maximum $|r[n]|$ is smaller than the threshold and the probability that the maximum $|r[n]|$ is bigger than the threshold with RTT estimation error, respectively.

\subsection{Comparison between IFDMA and LFDMA}

As stated in the previous section, IFDMA and LFDMA are originally proposed to utilize channel diversity gain and modulation gain, respectively. However, they are not directly applicable to multiple random access system since it is based on the peak detection. Fig. 3 illustrates the compassion between IFDMA and LFDMA in the multiple random access system. Number of random access subcarriers is set to 84 and five UEs are assumed to transmit random access signal simultaneously. Total transmit power of random access signal is normalized to 1 and Rural multipath fading channel is adopted.

Fig. 4a and $4 \mathrm{~b}$ show the PDF and the cumulative distribution function (CDF) of normalized $|r[n]|_{\text {Max }}$, which is defined in Eq. (12).

$$
|r[n]|_{\text {Max }}=\underset{\tilde{c}[n], \Delta l}{\arg \max }|r[n]| .
$$

For the same SNR, both PDF and CDF of LFDMA have rightshifted distribution than those of IFDMA, which refers that the peak of LFDMA is likely to be bigger than that of IFDMA. Statistically, the envelope of $r[n]_{\text {LFDMA }}$ is bigger than $r[n]_{\text {IFDMA }}$, which is also analytically derived in Appendix A. Thus, it is determined that LFDMA outperforms IFDMA in random access detection.

\subsection{Random Access Detection Miss Rate}

The threshold of detection miss is set to avoid RTT estimation error when random access signal power is weak. In case of detection miss, the Node B can not recognize the random access try of UE and does not transmit random access success message to UE. Thus, UE is able to know random access failure and it transmits random access signal again using the next random

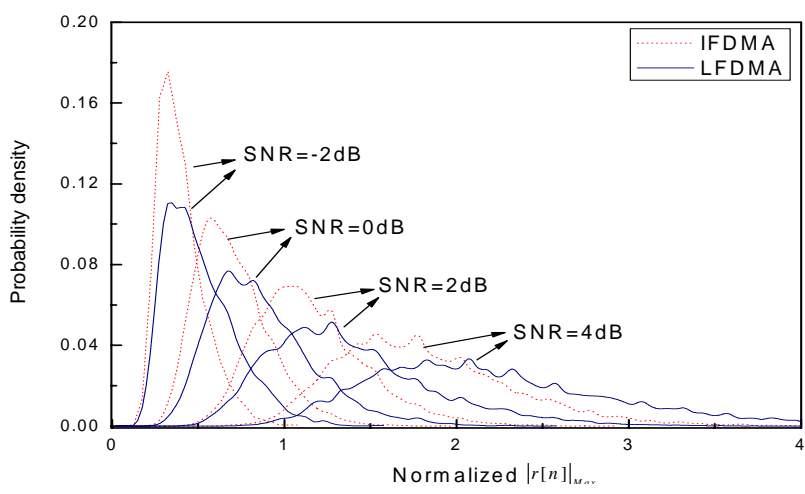

(a) PDF comparison.

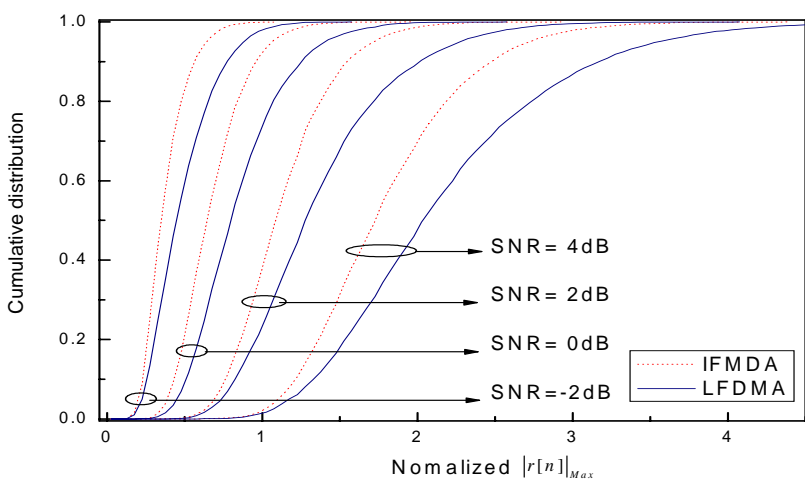

(b) CDF comparison.

Fig. 3 Comparisons of PDF and CDF of LFDMA and IFDMA.

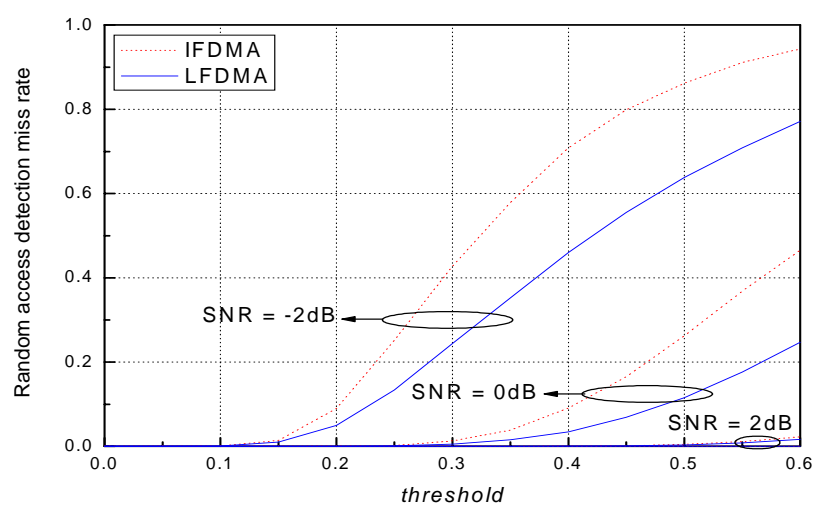

Fig. 4 Random access detection miss rate.

access opportunity. However, in case of RTT estimation error the Node B and UE continue further communication with wrong RTT estimation until they recognize the estimated RTT was incorrect. UE transmit a new random access signal again after it recognizes the RTT estimation error. Hence, it takes significant time for UE to transmit a new random access signal. 
$P_{M}$ mainly depends on SNR and the threshold provided that random access signal of multiple UEs have equal power. Fig. 4 shows $P_{M}$ of IFDMA and LFDMA with various threshold set when the number of UEs and random access subcarriers are 5 and 84, respectively. $\mathrm{X}$-axis and $\mathrm{Y}$-axis are the threshold and $P_{M}$. When SNR is bigger than $2 \mathrm{~dB} P_{M}$ is almost zero until the threshold equals to 0.6. However, $P_{M}$ becomes exceed 0.5 when the threshold is set to 0.4 with SNR of $-2 \mathrm{~dB}$. In this paper, we set the threshold to 0.3 in order to provide random access functionality at low SNR. In this case, $P_{M}$ is about 0.25 for LFDMA and 0.42 for IFDMA when SNR equals to $-2 \mathrm{~dB}$. For both LFDMA and IFDMA $P_{M}$ are close to zero when SNR is bigger than $0 \mathrm{~dB}$. From the Fig. 4, IFDMA is determined not suitable for random access in terms of $P_{M}$.

\subsection{RTT Estimation Error}

In multiple random access scenarios, the output of multiple access detection of random access code $i$ is described as Eq. (13) including other UEs' signals. As shown in Eq. (13), the performance of multiple access detection highly depends on the channel characteristics, interfering UEs, and noise. Among them, channel and interference causes significant effects to system performance since they are not overcome by power control.

Fig. 4 shows the comparison of $P_{E}$ among perfect channel, Rural multipath fading channel, and Urban multipath fading channel. 10000 times of simulations are performed with various numbers of subcarriers and interfering UEs. The threshold and SNR are set to 0.3 and $0 \mathrm{~dB}$, respectively. Discrepancy of RTT estimation exceeding 1.25 usec is set to RTT estimation error.

Fig. 4a illustrates $P_{E}$ of perfect channel. In this case $H_{m}\left(f_{k}\right)$ and $H_{m}\left(f_{Q k}\right)$ equal to one. Thus, it depicts the capability of the crosscorrelation property of Zadoff-Chu code. $P_{E}$ is almost zero in the whole region thanks to the superb correlation property of ZadoffChu code. Yet, some non-zero region is also found as number of interfering UEs increases.

Fig. 4b and 4c illustrate $P_{E}$ of Rural and Urban multipath fading channel with LFDMA system. It is easily confirmed that the performance of random access is degraded as the number of interfering UEs increases. It derives the fact that the correlation property of random access code is affected by the interference. It is also determined that $P_{E}$ decreases as the number of random access subcarriers increases with the same number of interfering UEs. Since the number of random access subcarriers refers the length of Zadoff-Chu code, the correlation property becomes strong against interference as code length increases. Thus, larger number of subcarriers shows the better performance. Meanwhile, trade-off between the performance and bandwidth efficiency exists. Comparing Fig. 4b and 4c, $P_{E}$ of the Rural channel shows superior results than that of Urban channel. As easily expected, longer RMS delay of the multipath profile causes more RTT estimation error.

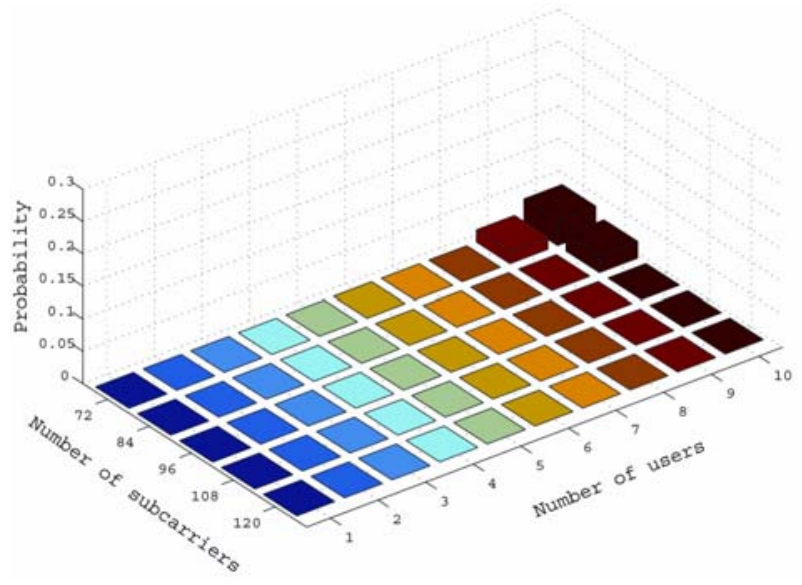

(a) Perfect channel.

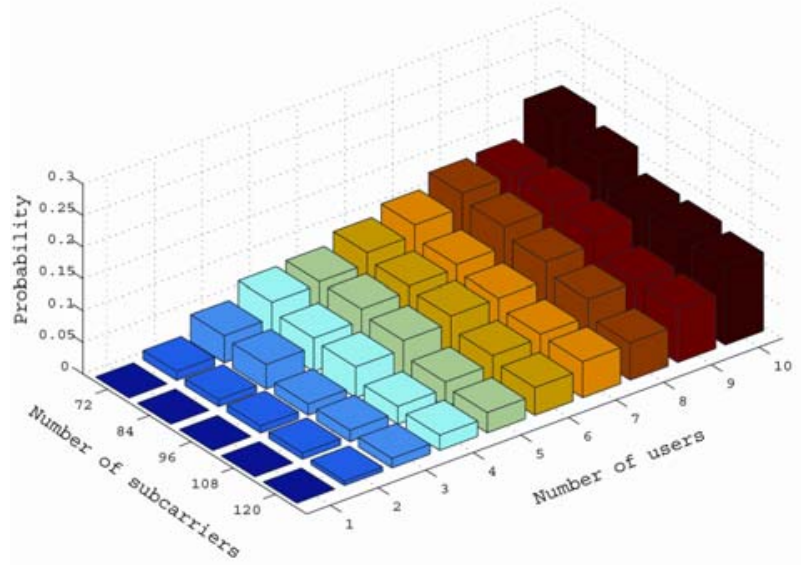

(b) Rural fading channel.

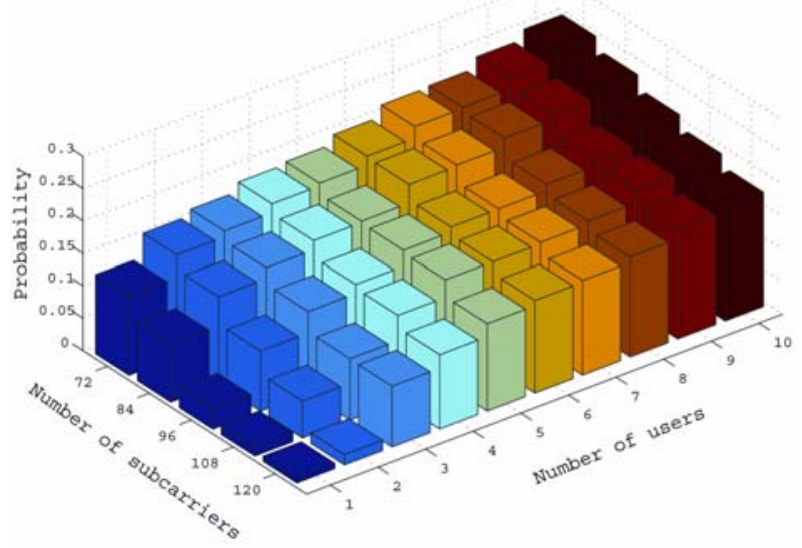

(c) Urban fading channel.

Fig. 5 Comparison of random access failure rate between perfect channel and urban fading channel.

\section{CONCLUSIONS}


In this paper, SC-FDMA random access system is analyzed. SCFDMA random access consists of synchronized and unsynchronized random access. We focused on the latter to investigate the performance of contention-based initial network entry process. In random access process, multiple UEs transmit randomly selected random access code. The multi-user resolution and uplink synchronization are achieved exploiting the crosscorrelation and auto-correlation of random access code, simultaneously. Considering the characteristics of single carrier system, we proposed the frequency domain RTT estimation and the time domain code identification. Random access detection shows the desired results in the perfect channel. However, it shows the performance degradation in the multipath fading channel. We analyzed the effect of multipath fading channel in terms of the number of interfering UEs and random access subcarriers. Moreover, we analytically proved that LFDMA outperforms IFDMA in random access. This paper will be the guideline in designing the SC-FDMA random access system.

\section{APPENDIX}

In this Appendix, envelopes of $r[n]_{L F D M A}$ and $r[n]_{I F D M A}$ in Eq. (13) will be analyzed. $r[n]$ in Eq. (13) is rewritten as Eq. (A.1) separating the desired signal from interferences and noise. When $m$ equals to zero it is assumed to the desired user.

$$
\begin{aligned}
& r[n]_{L F D M A}=r[n]_{L F D M A}^{\text {desired }}+r[n]_{L F D M A}^{\text {interference }}+n '[n], n=0,1, \ldots N-1, \\
& r[n]_{I F D M A}=r[n]_{I F D M A}^{\text {desired }}+r[n]_{I F D M A}^{\text {interference }}+n "[n], n=0,1, \ldots N-1,
\end{aligned}
$$

where $r[n]_{L F D M A}^{\text {desired }}, r[n]_{L F D M A}^{\text {interference }}, r[n]_{I F D M A}^{\text {desired }}$, and $r[n]_{I F D M A}^{\text {interference }}$ are given as

$$
\begin{aligned}
& r[n]_{L F D M A}^{\text {desired }}=\sum_{k=0}^{N-1} H_{0}\left(f_{k}\right), \quad r[n]_{I F D M A}^{\text {desired }}=\sum_{k=0}^{N-1} H_{0}\left(f_{Q k}\right), \\
& r[n]_{L F D M A}^{\text {interference }} \\
& =\sum_{m=1}^{I-1} \sum_{k=0}^{N-1}\left[c_{m}[n]\left(\widetilde{c_{0}}[n]\right)^{*} \cdot H_{m}\left(f_{k}\right) \cdot \exp \left\{j 2 \pi f_{k}\left(l_{o}^{R T T}-l_{m}^{R T T}\right)\right\}\right], \\
& r[n]_{I F D M A}^{\text {interence }} \\
& =\sum_{m=1}^{I-1} \sum_{k=0}^{N-1}\left[c_{m}[n]\left(\widetilde{c_{0}}[n]\right)^{*} \cdot H_{m}\left(f_{Q k}\right) \cdot \exp \left\{j 2 \pi f_{k}\left(l_{o}^{R T T}-l_{m}^{R T T}\right)\right\}\right] .
\end{aligned}
$$

In the Rayleigh multipath fading channel, in-phase and quadrature components of both $H_{0}\left(f_{k}\right)$ and $H_{0}\left(f_{Q k}\right)$ consist of zero-mean Gaussian random variable. Therefore, in-phase and quadrature components of $r[n]_{L F D M A}^{\text {desired }}$ and $r[n]_{\text {IFDMA }}^{\text {desired }}$ are also zero-mean Gaussian random variables because they are summation of $N$ zero-mean Gaussian random variables [16]. Likewise, in-phase and quadrature components of $r[n]_{L F D M A}^{\text {interference }}$ and $r[n]_{L F D M A}^{\text {interference }}$ are also zero-mean Gaussian random variables consisting of summation of $N \times(I-1)$ zero-mean Gaussian random variables. Although mismatched random access codes $c_{m}[n]\left(\widetilde{c_{0}}[n]\right)^{*}$ and uncompensated RTT estimation components $\exp \left\{j 2 \pi f_{k}\left(l_{o}^{R T T}-l_{m}^{R T T}\right)\right\} \quad$ and $\exp \left\{j 2 \pi f_{Q k}\left(l_{o}^{R T T}-l_{m}^{R T T}\right)\right\} \quad$ exist, they affect only the phase of $r[n]_{L F D M A}^{\text {interference }}$ and $r[n]_{L F D M A}^{\text {interference }}$ Moreover, in-phase and quadrature components of $n^{\prime}[n]$ and $\mathrm{n}$ "[n] are Gaussian random variable as well since they are summation of $N$ phase transformed AWGN. Thus, in-phase and quadrature components of $r[n]_{\text {LFDMA }}$ and $r[n]_{\text {IFDMA }}$ are regarded as Gaussian random variables and envelopes of them are modeled as Rayleigh random variables [16].

To obtain PDFs of Rayleigh random variables $|r[n]|_{\text {LFDMA }}$ and $|r[n]|_{\text {IFDMA }}$, variances of in-phase and quadrature components of $r[n]_{L F D M A}$ and $r[n]_{I F D M A}$ are necessary and sufficient. Variances of in-phase component of $r[n]_{L F D M A}^{\text {desired }}$ and $r[n]_{\text {IFDMA }}^{\text {desired }}$ are given as Eq. (A.3). Variances of quadrature component are abbreviated since they are identical to those of in-phase component.

$$
\begin{aligned}
& \operatorname{VAR}\left(r[n]_{L F D M A}^{\text {desired }, I}\right)=\sum_{k=0}^{N-1} \operatorname{VAR}\left(H_{0}^{I}\left(f_{k}\right)\right)+2 \sum_{i=0}^{N-1} \sum_{\substack{j=0 \\
j \neq 1}}^{N-1} \operatorname{COV}\left(H_{0}^{I}\left(f_{i}\right), H_{0}^{I}\left(f_{j}\right)\right), \\
& \operatorname{VAR}\left(r[n]_{I F D M A}^{\text {desired }, I}\right)=\sum_{k=0}^{N-1} \operatorname{VAR}\left(H_{0}^{I}\left(f_{Q k}\right)\right)+2 \sum_{i=0}^{N-1} \sum_{\substack{j=0 \\
j \neq 1}}^{N-1} \operatorname{COV}\left(H_{0}^{I}\left(f_{Q i}\right), H_{0}^{I}\left(f_{Q j}\right)\right),
\end{aligned}
$$

where $V A R, C O V$, and subscript $I$ refer variance, covariance, and in-phase component, respectively.

Statistically, averages of $\sum_{k=0}^{N-1} \operatorname{VAR}\left(H_{0}^{I}\left(f_{k}\right)\right)$ and $\sum_{k=0}^{N-1} \operatorname{VAR}\left(H_{0}^{I}\left(f_{Q k}\right)\right)$ are identical as given Eq. (A.4) since the channel impulse response is not biased to the specific frequency band in terms of time average.

$$
E\left[\sum_{k=0}^{N-1} \operatorname{VAR}\left(H_{0}^{I}\left(f_{k}\right)\right)\right]=E\left[\sum_{k=0}^{N-1} \operatorname{VAR}\left(H_{0}^{I}\left(f_{Q k}\right)\right)\right]
$$

where $E$ refers time average. On the other hand, averages of

$$
\begin{aligned}
& r[n]_{L F D M A}=\sum_{m=0}^{I-1} \sum_{k=0}^{N-1}\left[c_{m}[n]\left(\widetilde{c_{i}}[n]\right)^{*} \cdot H_{m}\left(f_{k}\right) \cdot \exp \left\{j 2 \pi f_{k}\left(l_{C P}-l_{m}^{R T T}-\Delta l\right)\right\}\right]+n^{\prime}[n], n=0,1, \ldots N-1, \\
& r[n]_{I F D M A}=\sum_{m=0}^{I-1} \sum_{k=0}^{N-1}\left[c_{m}[n]\left(\widetilde{c_{i}}[n]\right)^{*} \cdot H_{m}\left(f_{Q k}\right) \cdot \exp \left\{j 2 \pi f_{Q k}\left(l_{C P}-l_{m}^{R T T}-\Delta l\right)\right\}\right]+n^{\prime \prime}[n], n=0,1, \ldots N-1 .
\end{aligned}
$$




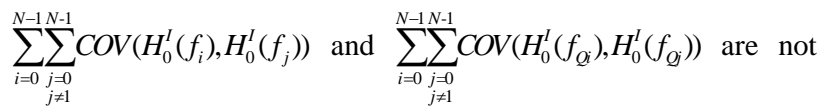
identical. Since $\operatorname{COV}\left(H_{0}^{I}\left(f_{a}\right), H_{0}^{I}\left(f_{b}\right)\right)$ has non-zero positive value only when subcarriers $f_{a}$ and $f_{b}$ are located within the coherence bandwidth, the average of $\sum_{i=0}^{N-1} \sum_{\substack{j=0 \\ j \neq 1}}^{N-1} \operatorname{COV}\left(H_{0}^{I}\left(f_{i}\right), H_{0}^{I}\left(f_{j}\right)\right)$ is statistically bigger than that of $\sum_{i=0}^{N-1 N-1} \sum_{\substack{j=0 \\ j \neq 1}}^{N} \operatorname{COV}\left(H_{0}^{I}\left(f_{Q_{i}}\right), H_{0}^{I}\left(f_{f_{j}}\right)\right)$ as given below.

$$
E\left[\sum_{i=0}^{N-1} \sum_{\substack{j=0 \\ j \neq 1}}^{N-1} \operatorname{COV}\left(H_{0}^{I}\left(f_{i}\right), H_{0}^{I}\left(f_{j}\right)\right)\right]>E\left[\sum_{i=0}^{N-1} \sum_{\substack{j=0 \\ j \neq 1}}^{N-1} \operatorname{COV}\left(H_{0}^{I}\left(f_{Q i}\right), H_{0}^{I}\left(f_{Q j}\right)\right)\right] .
$$

Although the situation varies depending on the coherence bandwidth, LFDMA will have more non-zero covariance pairs than IFDMA because adjacent subcarriers are allocated for random access. Hence, Eq. (A.6) holds true from Eq. (A.4) and Eq. (A.5).

$$
E\left[\operatorname{VAR}\left(r[n]_{L F D M A}^{\text {desired, } I}\right)\right]>E\left[\operatorname{VAR}\left(r[n]_{I F D M A}^{\text {desired, } I}\right)\right]
$$

Meanwhile, averages of variances of in-phase component of $r[n]_{L F D M A}^{\text {interference }}$ and $r[n]_{I F D M A}^{\text {interference }}$ are statistically identical as Eq. (A.7) because $H_{m}\left(f_{k}\right)$ and $H_{m}\left(f_{Q k}\right)$ are scrambled by mismatched random access codes.

$$
E\left[\operatorname{VAR}\left(r[n]_{L F D M A}^{\text {interference, } I}\right)\right]=E\left[\operatorname{VAR}\left(r[n]_{I F D M A}^{\text {interference, } I}\right)\right] .
$$

Like wise Eq. (A.8) holds true for noise components.

$$
E\left[\operatorname{VAR}\left(\mathrm{n}^{\prime}[n]^{I}\right)\right]=E\left[\operatorname{VAR}\left(\mathrm{n}^{\prime \prime}[\mathrm{n}]^{I}\right)\right]
$$

Finally, PDFs of $|r[n]|_{L F D M A}$ and $|r[n]|_{\text {IFDMA }}$ are given as below

$$
\begin{aligned}
& f_{\mid r\left[n_{L F D M A} \mid\right.}\left(x_{L F D M A}\right)=\frac{x_{L F D M A}}{\sigma_{\left|r[n]_{L F D M A}\right|}^{2}} \exp \left(\frac{-x_{\text {LFDMA }}^{2}}{2 \sigma_{\left|r[n]_{L \text { LDMM }}\right|}^{2}}\right), \quad\left(0 \leq x_{\text {LFDMA }}<\infty\right), \\
& f_{\left|r[n]_{[F D M M A}\right|}\left(x_{I F D M A}\right)=\frac{x_{I F D M A}}{\sigma_{\mid\left[[n]_{I F D M A} \mid\right.}^{2}} \exp \left(\frac{-x_{I F D M A}^{2}}{2 \sigma_{\left|r[n]_{I F D M A}\right|}^{2}}\right),\left(0 \leq x_{I F D M A}<\infty\right),
\end{aligned}
$$

where $\sigma_{\mid\left[[n]_{L F D M A} \mid\right.}^{2}$ and $\sigma_{\left|r[n]_{\text {IFDMA }}\right|}^{2}$ are defined as

$$
\begin{aligned}
& \sigma_{\left|r[n]_{\text {LDDMa }}\right|}^{2}=\operatorname{VAR}\left(r[n]_{L F D M A}^{\text {desired, } I}\right)+\operatorname{VAR}\left(r[n]_{L F D M A}^{\text {intereferece,I }}\right)+\operatorname{VAR}\left(\mathrm{n}^{\prime}[n]^{I}\right), \\
& \sigma_{\mid\left[r[]_{[F D M A} \mid\right.}^{2}=\operatorname{VAR}\left(r[n]_{I F D M A}^{\text {desired, } I}\right)+\operatorname{VAR}\left(r[n]_{I F D M A}^{\text {interference, } I}\right)+\operatorname{VAR}\left(\mathrm{n}[n]^{I}\right) \text {. }
\end{aligned}
$$

From Eq. (A.6), (A.7), (A.8), and (A.10) $\sigma_{\left|r[n]_{L F D M A}\right|}^{2}$ is determined to be bigger than $\sigma_{\mid\left[[n]_{\text {IFDMA }} \mid\right.}^{2}$ in terms of time average. This refers that PDF of LFDMA will have right-shifted distribution than those of IFDMA and it corresponds to Fig. 4a.

\section{REFERENCES}

[1] 3GPP, TR 25.814 V7.1.0, "Physical layer aspect for evolved Universal Terrestrial Radio Access (UTRA),” Sep. 2006.

[2] 3GPP, TSG R1-050591, NTT DoCoMo, "Physical Channels and Multiplexing in Evolved UTRA Uplink,” Jun. 2005.

[3] 3GPP, TSG R1-051445, Ericsson, "E-UTRA random access,” Nov. 2005.

[4] H.G. Myung, et al. "Single carrier FDMA for uplink wireless transmission,” IEEE Veh. Mag. vol. 1, no. 3, pp.30-38, Sep. 2006.

[5] D. Falconer, et al. "Frequency domain equalizer for singlecarrier broadband wireless systems” IEEE Comm. Mag. Vol. 40, no. 4, pp. 58-66, Apr. 2002.

[6] H.A. Mahmoud and M.K. Ozdemir, "Initial ranging for WiMAX (802.16e) OFDMA,” in Proc. IEEE Military Communications Conference 2006, Oct. 2006.

[7] H. Minn and X. Fu, "A new ranging method for OFDMA systems," in Proc. IEEE Global Communications Conference 2005, Dec. 2005.

[8] M. Morelli, "Timing and frequency synchronization for the uplink of an OFDMA system," IEEE Trans. Comm., vol. 52, no. 2, pp. 296-306, Feb. 2004.

[9] E. Bala and L.J. Cimini, Jr. "On the uplink synchronization of OFDMA system," in Proc. IEEE Military Communications Conference 2005, Oct. 2005.

[10] Z. Ma and Y.I. Kim, "A time-frequency synchronization algorithm free of ISI for HPI system," in Proc. IEEE Vehicular Technology Conference 2004 Fall, Nov. 2004.

[11] L.Lu and V.K. Dubey, "Extended orthogonal polyphase codes for multicarrier CDMA system," IEEE Comm. Lett. vol. 8, no. 12, pp. 700-702, Dec. 2004.

[12] B.M.Popovic, "Generalized chirp=like polyphase sequences with optimum correlation properties," IEEE Trans. on Information Theory. vol.38, no. 6, pp. 1406-1409, Jul. 1992.

[13] W.H. Mow and S.-Y.R. Li, "Aperiodic autocorrelation properties of perfect polyphase sequences," in Proc. IEEE ICCS/ISITA’92, 1992.

[14] IMA111-0E, "UMTS long term evolution (LTE) technology introduction,” Rohde \& Schwarz, 2007. 
[15] K. Jeong, et al. "Multipath channel models for wireless local and metropolitan area networks," in Proc. IEEE Third International Conference on Information Technology and Applications, vol. 2, pp. 295-298, Jul. 2005.

[16] A. Leon-Garcia, Probability and Random Processes for Electrical Engineering, Addison Wesley Inc., 1994. 